
UNDER CONTRACT DE-AC02-76-CHO-3073

PPPL-2817

UC-420,427

\title{
NONLINEAR RELATIVISTIC INTERACTION \\ OF AN ULTRASHORT LASER PULSE WITH A COLD PLASMA
}

BY

J.M. RAX AND N.J. FISCH

January 1992

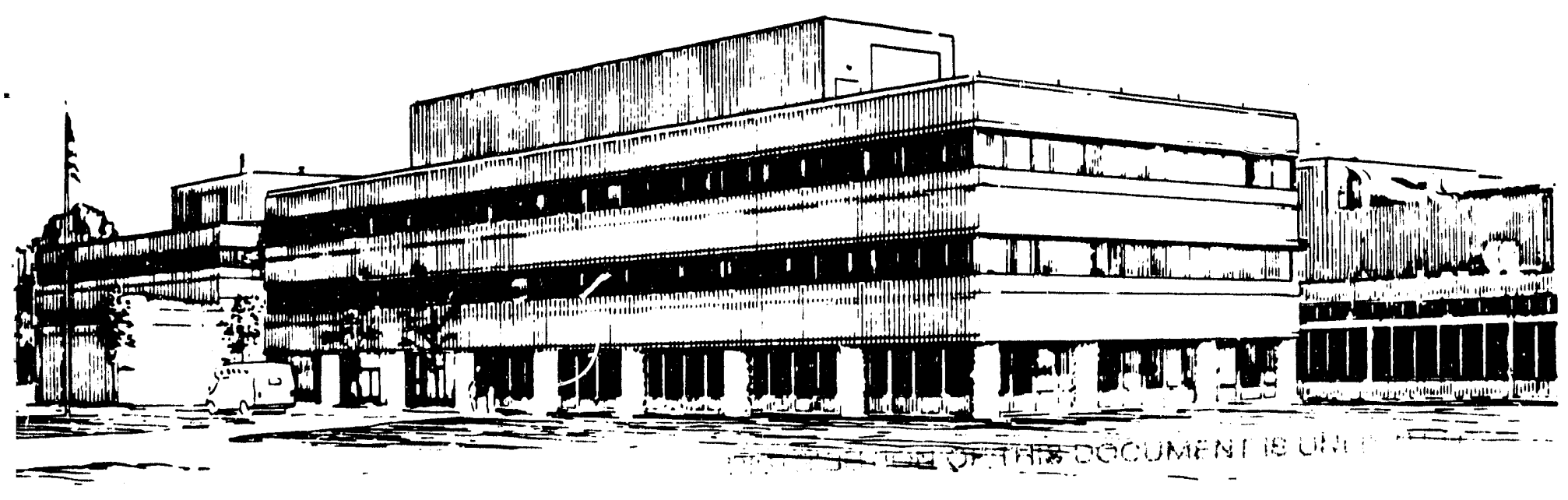




\section{NOTICE}

This report was prepared as an account of work sponsored by an agency of the United States Government. Neither the United States Government nor any agency thereof, nor any of their employees, makes any warranty, express or implied, or assumes any legal liability or responsibility for the accuracy, completeness, or usefulness of any information, apparatus, product, or process disclosed, or represents that its use would not infringe privately owned rights. Reference herein to any specific cornmercial produce, process, or service by trade name, trademark, manufactureir, or otherwise, does not necessarily constitute or imply its endorsement, recommendation, or favoring by the United States C overnment or any agency thereof. The views and opinions of authors expressed here in do not necessarily state or reflect those of the United States Government or any agency thereof.

\section{NOTICE}

This report has been reproduced directly from the best available copy.

Available to DOE and DOE contractors from the:

Office of Scientific and Technical Information

P.O. Box 62

Oak Ridge, TN 37831;

Prices available from (615) 576-8401.

Available to the public from the:

National Technical Information Service

U.S. Department of Commerce 5285 Port Royal Road

Springfield, Virginia 22161

703-487-4650 


\title{
Nonlinear relativistic interaction of an ultrashort laser pulse with a cold plasma
}

\author{
J.M. Rax*and N.J. Fisch \\ Princeton Plasma Physics Laboratory, \\ Princeton University, Princeton, NJ 08543
}

\begin{abstract}
We investigate the nonlinear, relativistic dynamics that result when intense $\left(10^{18} \mathrm{~W} / \mathrm{cm}^{2}\right.$ and above) and ultrashort (one plasma period or shorter) laser pulse travels through a cold underdense plasma. Using a Lagrangian analysis of the plasma reponse, it can be demonstrated that the nonlinear wake. the collective dissipation, the nonlinear Compton losses, and the harmonic generation, are all determined by a finite set of integrated scalar quantities. This result holds for one-dimensional, short pulses of arbitrary amplitude, shape, and polarization, so that these very short intense laser pulses in a plasma can be viewed essentially as a quasiparticle characterized by a small set of global parameters.
\end{abstract}

- Permanent adress: Association EURATOM-CEA, C.E.N Cad., 1310\& St Paul lez Durance. FRA.VCE. 


\section{Introduction}

That electrons or photons might be accelerated by high phase velocity nonlinear plasma waves [1] focusses attention to the possibility that such waves might be generated by means of intense ultrashort laser pulses in a cold plasma. Techniques of pulse compression [2] now make possible the exploration of the laser-plasma interaction at fluxes above $10^{18} \mathrm{~W} / \mathrm{cm}^{2}$. Thus, there is a need to develop new theoretical tools to understand the intense relativistic pulse-plasma interaction regime.

The nonlinearity parameter of an electromagnetic transvese wave with vector potential $a$ is $\eta=e a / m c$ where $c$ is the velocity of light, $-e$ the electron chage, and $m$ the electron mass [3]. When $\eta \sim 1\left(\sim 10^{18} \mathrm{~W} / \mathrm{cm}^{2}\right.$ for visible light $)$, the quiver velocity becomes relativistic. When the fields are so strong that the nonlinearity parameter $\eta>1$, the dipolar approximation is no longer valid, the Lorentz equations becomes nonlinear because of the occurrence of a space-dependent term in the driving wave phase, and the relativistic momentum is a nonlinear function of the velocity. Yet another source of nonlinearity, appearing in the Eulerian representation but avoided in a Lagrangian analysis, arises from the convective derivative of the velocity.

This paper addresses the problem of the nonlinear relativistic interaction of a given one-dimensional, very short, electromagnetic pulse of arbitrary amplitude, polarization and shape, with a cold underdense plasmas. The plasma is considered cold, in that both the electron quiver velocity in the pulse, and the electron longitudinal velocity in the wake, are larger than the electron thermal velocity.

Although there are no general methods to deal with such a nonlinear, initial value problem, a Lagrangian analysis of the electrons plasma response is powerful mathematically, and affords a clear physical pictures of the nonlinear processes. Here we shall consider the effects of a given pulse on the plasma; the effects of the plasma on the pulse occur on a time scale longer than the times scales involved in the dynamics of the plasma responses. The slow evolution of the shape of the pulse will be considered in a forthcoming paper.

The consideration of an ultrashort pulse actually simplifies consiclerably the mathematical analysis. This is fortunate, since, in the relativistic regime, the dipolar expansion which assumes $\eta \ll 1$ can not be used. Moreover, the usual formalism of parametric coupling of nonlinear laser-plasma interaction s can not be put at work efficiently, because, in he regime we consider, the plasma experiences a ballistic, very short, passing perturbation rather than an harmonic one.

Consider an ultrashort pulse with a broad spectra whose mean frequency, $\bar{\omega}$, is above the plasma frequency, $\omega_{p}$, and whose width, $\delta \omega$, is larger than $\omega_{p}$. The plasma is assumed to be underdense for the main spectral components of the pulse $\left(\bar{\omega} \pm \delta \omega>\omega_{p}\right)$. Thus, there are two small parameters, $\omega_{p} / \bar{\omega}<1$, and $\omega_{p} / \delta \omega<1$, and, since $\delta \omega<\bar{\omega}$, the second inequality ensures the first one.

The inequality $\omega_{p} / \delta \omega<1$ means that the pulse duration is shorter than the time for the electrons to set up a collective response. In other words, the electron motion inside 
the pulse is dominated by the single particle response to the transverse wave packet, and the collective longitudinal response can be treated as a perturbation in front of the driving pulse forces. On the other hand, behind the pulse the plasma reorgnizes itself through a purely longitudinal collective response to the initial perturbation induced by the pulse, and this result in an electrostatic wake.

A Lagrangian analysis, efficient in analysing the weakly relativistic regime of the beat wave problem, [4] is also suited particularly to the present parameter regime of a given arbitrary short pulse on a cold plasma. This problem of intense relativistic pulse-plasma interaction has been recently addressed with a quasistatic approximation of the Eulerian representation [5], which assumes an ordering of the same type. However, using present Lagrangian method, rather than the quasistatic approximation of the Eulerian representatation, it can be shown that the plasma responses (wake, harmonic, Compton diffusion) can be calculated in terms of a small set of global quantities; moreover, this can be accomplished for arbitrary amplitude, shape and polarization, of the pulse. From this analysis, the pulse appears as a quasiparticle characterized by a few scalars parameters, which we calculate. The Lagrangian analysis provided here can also deal with discreteness effects, such as nonlinear Compton losses which do not appear in the fluid representation.

Suppose a wave packet propagates in the $z$ direction, with vector potential $a(t-z / c)$. Suppose further that $\mathbf{a}(t-z / c<0)=\mathbf{a}(t-z / c>T)=0$. The pulse is then characterized by two time scales: its mean frequency $\bar{\omega}$ and its total phase duration $T$, where $T \delta \omega \sim 1$. Our study is restricted to a pulse traveling at the velocity of light, which is a good approximation for waves in an underdense plasma, and an even better approximation for very intense waves [6]. In that case, the duration seen by a rest observer is, in fact, $T \sqrt{2}$.

It turns out that, for short pulses, many important phenomena do not depend on the details of the pulse shape; rather, certain global parameters play a key role. For example, consider the total energy content per unit surface, $u$. This quantity can be expressed in term of the square of the electric field $\left(\dot{a}^{2}=\dot{\mathbf{a}} \cdot \dot{\mathbf{a}}\right)$ as follows:

$$
u \equiv \epsilon_{0} U \equiv \epsilon_{0} \int_{0}^{T} \dot{a}^{2}(u) d u \text {. }
$$

As will be demonstrated in the forthcoming sections, a set of integrated quantities, or what we call "global parameters," of which $U$ is a member, turns out to characterize the plasma dynamical responses.

The paper is organized as follows: In Sec. 2 and Sec. 3 we review the exact relativistic orbit of an electron in an arbitrary electromagnetic pulse, and the Lagrangian theory of relativistic nonlinear plasma waves. In Sec. 4 we study the relativistic interaction of a short, intense pulse with an underdense plasma. The electron response inside the pulse is calculated through an expansion that exploits the exact calculation, discussed in Sec. 2, of electron motion in vacuum fields. The net effect of the pulse on the plasma depend: on two quantities that describe the electron as it leaves the pulse, the exit position $H$, and the exit velocity $V$. These quantities, like $U$ above, can be expressed as weighted integrals over the pulse. 
In Sec. 5, and Sec. 6, on the basis of $H$ and $V$, the wake structure, and collective energy losses, are calculated. Then in Sec. 7 and Sec. 8, nonlinear Compton losies and harmonic generation are studied, and these effects are shown to be determined by two new global parameters. A Lagrangian picture of photon acceleration is briefly analyzed in Sec. 9. In Sec. 10, the various density regimes for nonlinear dissipation are explored. In Sec. 11, our results and conclusions are summarized.

To simplify the presentation, in the following, rather than the I.S. of units we shall use $m=c=e=1$. Thus, the nonlinearity parameter $\eta$ is in fact $a$, and the permittivity of free space $\epsilon_{0}$ is the inverse of the clasical electron radius $1 / 4 \pi r_{e}$.

\section{Exact relativistic motion in a laser pulse}

The relativistic motion of an electron in an electromagnetic pulse, of arbitrary polarization and shape is integrable [7]. In this section, we will briefly review this important result which is the underpinning of the Lagrangian analysis. Integrability is a consequence of the existence of a space-time symmetry associated with the phase of the wave: since the system is invariant with respect to translation along the phase direction in space-time, Noether's theorem assures the existence of an additional invariant associated with this symmetry. The motion of the electron in the wave is described by the the Lorentz equation,

$$
\frac{d \mathrm{p}}{d t}=\dot{\mathrm{a}}+\mathrm{v} \times(\mathrm{n} \times \dot{\mathrm{a}}), \quad \frac{d \gamma}{d t}=\dot{\mathrm{a}} \cdot \mathrm{v},
$$

where the wave travels in the direction of the unitary vector $n$, the electron momentum is denoted by $\mathrm{p}$ and the velocity by $\mathrm{v}$. The dot stands for differentiation with respect to the phase argument, $(t-z)$, and $\gamma$ is the relativistic energy. Multiplying the first equation by $\mathbf{n}$, using $\mathbf{a} \cdot \mathbf{n}=0$, and subtracting the second equation, we find that the quantity $\gamma-\mathbf{n} \cdot \mathbf{p}$ is a constant of the motion. In a cold plasma $\left(T_{e} \ll 511 \mathrm{KeV}\right)$, this constant is clearly $\gamma(-\infty)-\mathbf{n} \cdot \mathrm{p}(-\infty)=1$. Thus, we have

$$
\gamma-\mathrm{n} \cdot \mathrm{p}=1, \quad \tau=t-z,
$$

where $\tau$ is the particle proper time, and the equation at right is simply a proper time integration (with a suitable choice of the integration constant) of the equation at left, i.e., $\gamma=d t / d \tau, \mathbf{n} \cdot \mathbf{p}=d z / d \tau$. The solution of Eq. 2 can then be expressed in term of this proper time as follows:

$$
z(\tau)=\frac{1}{2} \int_{0}^{\tau} a^{2}(u) d u, \quad \gamma(\tau)=1+\frac{1}{2} a^{2}(\tau),
$$

where we have used the conservation of the canonical transverse momentum, $\mathrm{n} \times \mathrm{p}=$ $\mathrm{n} \times \mathbf{a}$. This implicit result, if not given explicitly, is, however, exact to all orders in a. Alternatively, we can express this implicit solution in the form,

$$
t(\tau)=\tau+\frac{1}{2} \int_{0}^{\tau} a^{2}(u) d u
$$


from where we can deduce the following physical interpretation: as the pulse passes the electron, no final exchange of energy or momentum between the pulse and the particle takes place, and the only effect of the wave packet on the electron after the packet passes the electron is a relativistic ponderomotive displacement of the electron, $\delta z=$ $\int_{0}^{T} a^{2}(u) d u / 2$, which occurs precisely in the direction of the wave propagation.

In a plasma, this displacement will induce electric forces resulting from perturbation of the charge density. The plasma will try to restore local charge neutrality. The competition between this plasma collective response and the pulse-induced ponderomotive displacement dominates the physics of nonlinear short pulse-plasma interaction inside the pulse. Clearly, however, from this picture we can anticipate certain excellent approximations, namely, that the main result of the pulse is a displacement, and subsequent dynamics can be considered without regard to the pulse.

\section{Exact relativistic motion in intense plasma waves}

We consider a cold plasma perturbed in the $z$-direction. Each electron is described by its unperturbed position $z_{0}$ and by its Lagrangian displacement $h\left(t, z_{0}\right)$, so that the running Eulerian position is given by $z=z_{0}+h[8]$. Assuming that the initial perturbation and the subsequent dynamics does not invert the initial $z_{0}$ ordering of the electrons (no overtaking), we can apply Gauss's theorem to find the relativistic motion along the $z$ axis:

$$
\frac{d p}{d t}=-\omega_{p}^{2} h, \quad \frac{d \gamma}{d t}=-\omega_{p}^{2} h \cdot \frac{d h}{d t} .
$$

Integrating the equation at right with respect to time, we find that $\gamma+\omega_{p}^{2} h^{2} / 2=1+$ $\omega_{p}^{2} h_{M}^{2} / 2$ is an invariant, where $h_{M}$ is the maximum elongation of the considered oscillation. This allows us to introduce the proper time $\tau$, and to express the dynamics in the proper time representation:

$$
\frac{d^{2} h}{d \tau^{2}}+\omega_{p}^{2}\left[1+\frac{\omega_{p}^{2} h_{M}^{2}}{2}\right] h-\frac{\omega_{p}^{4}}{2} h^{3}=0
$$

Thus, the proper time representation of the dynamics leads to a nonlinear oscillator equation, whose solution can be put analytically in terms of Jacobian elliptic functions:

$$
h(\tau)=h_{M} \mathrm{sn}(\omega \tau, k), \quad t(\tau)=\tau-\frac{K(k)}{\omega}+\omega_{p}^{2} \frac{h_{M}^{2}}{2} \int_{\frac{K(k)}{\omega}}^{\tau} \mathrm{cn}^{2}(\omega u, k) d u,
$$

where $\omega$ and $k$ are, in fact, functions of the amplitude, given by

$$
\omega^{2}=\omega_{p}^{2}\left[1+\ddot{\omega}_{p}^{2} \frac{h_{M}^{2}}{4}\right], \quad k^{2}=\frac{\omega_{p}^{4} h_{M}^{2}}{4 \omega^{2}}
$$

The initial condition is $h(t=0)=h_{M}$, and $K^{\prime}$ is the complete elliptic integral of the first kind. It is to be noted that the integral of $\mathrm{cn}^{2}$ in Eq. 8 can be expressed in terms of the 
elliptic integral of the second kind $E$, i.e., $k^{2} \int^{u} \operatorname{cn}^{2}[v, k] d v=E[\operatorname{am}(u), k]-\left(1-k^{2}\right) u$. As for the solution in the pulse, but without the plasma, described in the previous section, the solution here is fully relativistic, and exact to all order in $h_{M}$, but implicit.

The nonlinear oscillator described by Eq. 7 can also be approached through perturbative methods; the well known result of such an analysis is relevant to the weakly relativistic regime and is given by:

$$
h=h_{M} \cos (\omega t), \quad \omega=\omega_{p}\left[1-3 \omega_{p}^{2} h_{M}^{2} / 16\right] .
$$

We shall now address the problem of the competition between the motion described in Secs. 2 and 3 , the regine in which the electron is inside a relativistic wave packet that propagates in a cold underdense plasma. The result of this competition will be captured by the parameters $H$ and $V$, the exit position and exit velocity from the pulse. Then, behind the pulse, the motion is described by Eqs. 8, with the initial conditions being $H$ and $V$.

\section{Pulse-plasma interaction}

To address the problem of the motion of a plasma electron first inside a pulse, and then behind it, let us refer to the space-time diagrams in Figs. 1 and 2. The forward and backward fronts of the pulse travel along two light characteristics, and the length of the pulse is $T$. When an electron enters the pulse, it is deflected according to the equations of motion as given by Eq. 2. Fig. 1 coresponds to the case of circular polarization and Fig. 2 corresponds to the case of linear polarization of the wave. This deflection causes a density perturbation inside the pulse, which leads to a longitudinal electric field. This collective electrostatic field tends to pull the particle back to its unperturbed position. Accordingly, we have

$$
\frac{d^{2} h}{d \tau^{2}}=\mathrm{n} \cdot[\mathbf{a} \times(\mathrm{n} \times \dot{\mathbf{a}})]-\omega_{p}^{2} \gamma h=\frac{\dot{a}^{2}}{2}-\omega_{p}^{2} \gamma h .
$$

The initial condition is $h=0$. If the pulse is short enough, the second term on the right hand side will remain smaller than the first term, so that we can expand about the exact result of Sec. 2, namely, we can expand $h=h_{0}+h_{1}+h_{2}+\ldots$. The other dynamical quantities of the problem can be expanded similarly, $\gamma=\gamma_{0}+\gamma_{1}+\gamma_{2}+\ldots$ ald so on. The first order correction, $h_{1}$, due to the presence of plasma, will be either of order $\omega_{P}^{2} / \omega^{2}$, $\omega_{P}^{2} / \delta \omega^{2}$, or $\omega_{P}^{2} / \omega \delta \omega$. As discussed in the ordering of Sec. 1 , these are, by assumption, small parameters; similarly, the $h_{2}$ term scales as $\omega_{p}^{4}$, etc. The system of equations to be solved is:

$$
\begin{aligned}
& \frac{d p}{d \tau}=\dot{a}^{2}(t-h) / 2-\omega_{p}^{2} h \gamma \\
& \frac{d \gamma}{d \tau}=\dot{a}^{2}(t-h) / 2-\dot{\omega}_{p}^{2} h p
\end{aligned}
$$




$$
\begin{aligned}
& \frac{d h}{d \tau}=p \\
& \frac{d t}{d \tau}=\gamma
\end{aligned}
$$

The zeroth order response is the one found in Sec. 2. The invariant, $\gamma_{0}-p_{0}=1$, allows to calculate the proper time, $\tau=t_{0}-h_{0}$, and the zeroth order energy and position

$$
h_{0}=\frac{1}{2} \int_{0}^{\tau} a^{2}(u) d u, \quad \gamma_{0}=1+\frac{1}{2} a^{2}(\tau) .
$$

The first plasma correction is governed by the system of equations

$$
\begin{aligned}
& \frac{d p_{1}}{d \tau}=\left(t_{1}-h_{1}\right) \ddot{a}{ }^{2}\left(t_{0}-h_{0}\right) / 2-\omega_{p}^{2} h_{0} \gamma_{0}, \\
& \frac{d \gamma_{1}}{d \tau}=\left(t_{1}-h_{1}\right) \ddot{a}\left(t_{0}-h_{0}\right) / 2-\omega_{p}^{2} h_{0} p_{0}, \\
& \frac{d h_{1}}{d \tau}=p_{1}, \\
& \frac{d t_{1}}{d \tau}=\gamma_{1} .
\end{aligned}
$$

To solve this system of equations, we subtract the first equation from the second one to derive an expression for $\gamma_{1}-p_{1}$. Then, we can solve for the quantity $t_{1}-h_{1}$, using

$$
\gamma_{1}-p_{1}=\omega_{p}^{2} \int_{0}^{\tau} h_{0}(s) d s, \quad t_{1}-h_{1}=\omega_{p}^{2} \int_{0}^{\tau} d u \int_{0}^{u} h_{0}(s) d s .
$$

With these expressions for the first order quantities, Eq. (17) can be solved explicitly. After integrating twice by parts, we get

$$
p_{1}=\frac{\omega_{p}^{2}}{4}\left[\dot{a}^{2} \int_{0}^{\tau} d u \int_{0}^{u} d v \int_{0}^{v} d s a^{2}(s) \cdots a^{2} \int_{0}^{\tau} d u \int_{0}^{u} d v a^{2}(v)-2 \int_{0}^{\tau} d u \int_{0}^{u} d v a^{2}(v)\right] .
$$

After further algebra, the total effect of the pulse on an electron can be expressed as a net displacement $H$ and as a small exit velocity $V$. The displacement, $H=h_{0}(T)+h_{1}(T)$, is illustrated schematically in Figs. 1 and 2. Note that the exit velocity, $V=p_{0}(T)+$ $p_{1}(T) /\left(\gamma_{0}(T)+\gamma_{1}(T)\right)=p_{1}(T)$, is a pure plasma effect. These exit quantities can be written as

$$
\begin{gathered}
H=\frac{1}{2} \int_{0}^{T} a^{2}(u) d u-\frac{1}{2} \omega_{p}^{2} \int_{0}^{T} d s \int_{0}^{s} d u \int_{0}^{u} d v a^{2}(v)\left[1+a^{2}(s)\right], \\
V=-\frac{1}{2} \omega_{p}^{2} \int_{0}^{T} d u \int_{0}^{u} d v a^{2}(v) .
\end{gathered}
$$

From this solution, we can define precisely the range of validity of the Lagrangian expansion. When an electron exits th pulse, its displacement, due to the plasma collective 
effect, must be smaller than the displacement due to the ponderomotive force described in Sec. 2, i.e., $h_{0}(T)>h_{1}(T)$. In the weakly relativistic regime, $a \sim 1$, this condition is equivalent to $\omega_{p}^{2}<\delta \omega^{2}$. In the strongly relativistic regime, $a \gg 1$, regime this condition is equivalent to $\omega_{p}^{2} a^{2}<\delta \omega^{2}$.

A complemeittary case, where the effect of a pulse on a plasma can be calculated analytically, is when the time ordering condition is relaxed, but the interaction is nonrelativistic, ( $a \ll 1)$. Admittedly, for the purpose of wake and harmonic generation, this nonrelativistic case is less important, but we present it here, for academic reasons, for the sake of completeness. Consider an electromagnetic pulse described by its vector potential $\mathbf{a}(t, z) \ll 1$. We do not assume the pulse length or the wave period to be smaller than the plasma wave pcriod, nor do we assume the group velocity to be the light velocity. In this linear, nonrelativistic case, $a$ is the small parameter. The Lagrangian coordinates of an electron inside the pulse are described by

$$
\frac{d^{2} h}{d t^{2}}=-\mathbf{n} \cdot\left[\mathbf{a}(t, z) \times\left(\mathbf{n} \times \frac{\partial \mathbf{a}(t, z)}{\partial z}\right)\right]-\omega_{p}^{2} h=-\mathbf{a}(t, z) \cdot \frac{\partial \mathbf{a}(t, z)}{\partial z}-\omega_{p}^{2} h .
$$

This equation is valid provided that the velocity of the electron remains smaller than the velocity of light. This occurs if $a \ll 1$, otherwise proper time corrections are needed. We can then use a dipolar approximation for the first term on the right hand side of Eq. 16, $\mathbf{a}(t, z)=\mathbf{a}\left(t, z_{0}\right)$, and $\partial \mathbf{a}(t, z) / \partial z=\partial \mathbf{a}\left(t, z_{0}\right) / \partial z$. Thus, the equation to be solveci becomes linear, and can be integrated directly. Let $T$ be the pulse length. The sole effect of the puise on the plasma, as in the previous case, is to disturb the electron position and velocity by an amount $H$ and $V$ given by

$$
H=\frac{1}{2 \omega_{p}} \int_{0}^{T} \sin \left[\omega_{p}(u-T)\right] \frac{\partial a^{2}(u, 0)}{\partial z} d u, \quad V=-\frac{1}{2} \int_{0}^{T} \cos \left[\omega_{p}(u-T)\right] \frac{\partial a^{2}(u, 0)}{\partial z} d u .
$$

Whereas from either a practical, or a fundamental, point of view, this nonrelativistic result may be less interesting than the relativistic one, this solution does arouse some interest. Note that, if $\omega_{p} T>1$, the scalings, with respect to $\omega_{p}$, and $a$, of the incremental displacement and velocity, $H$ and $V$, are different from the fully relativistic nonlinear case. This-indicates that to reach perturbatively, from the nonrelativistic case, the relativistic case may require a large number of terms in the expansions that rely upon the smallness of $\omega_{p}$ or $a$.

\section{Nonlinear wake}

Using the results of the previous sections, the structure of the nonlinear wake betnind the pulse can be calculated easily. First, let us investigate the weakly relativistic response described by Eq. (10). The weakly relativistic Lagrangian displacement behind the pulse can be written as

$$
h\left(t, z_{0}\right)=H \cos \left[\omega\left(t-z_{0}\right)\right]+\frac{V}{\omega} \sin \left[\omega\left(t-z_{0}\right)\right], \quad t>z_{0},
$$


where $z_{0}$ is the initial unperturbed position of the electron. The nonlinear frequency is given by

$$
\omega(H, V)=\omega_{p}\left(1-3 \omega_{p}^{2} h_{M}^{2} / 16\right), \quad h_{M}^{2}=H^{2}+\frac{V^{2}}{\omega_{p}^{2}} .
$$

It can be verified that Eq. (27) fulfills both the dynamical equation of Sec. 3 and the initial condition just behind the pulse calculated in Sec. 4. The Eulerian density perturbation can be expressed on the basis of the unperturbed density $n\left(z_{0}\right)$ and $h$, namely

$$
n(z, t)=\int d z_{0} n\left(z_{0}\right) \delta\left[z-z_{0}-h\left(t, z_{0}\right)\right]=\int d z_{0} \int \frac{d k}{2 \pi} n\left(z_{0}\right) e^{-i k\left[z-z_{0}-h\left(t, z_{0}\right)\right]}
$$

where $\delta$ is the Dirac function. The exponential of the oscillating Lagrangian position in Eq. (27) can be expanded in terms of Bessel functions of the first kind, $J_{n}$. For $t>z$, we obtain, behind the pulse,

$$
n=\int_{-\infty}^{t} d z_{0} \int_{-\infty}^{+\infty} \frac{d k}{2 \pi} \sum_{m=-\infty}^{m=+\infty} \sum_{n=-\infty}^{n=+\infty} i^{m} n\left(z_{0}\right) J_{n}(k V / \omega) J_{m}(k H) e^{-i k\left(z-z_{0}\right)} e^{i(m+n) \omega\left(t-z_{0}\right)}
$$

Note that after at least one plasma period, $h\left(z_{0}, t\right)$ is unaffected by the electrons just leaving the pulse. Thus, the $z_{0}$ integral can be extended from $-\infty$ to $+\infty$. It then becomes apparent that Eq.(30) simplifies, because the integral over $z_{0}$ can be performed, giving the the Fourier transform of the unperturbed density profile $n\left(z_{0}\right)$. Thus, Eq.(30) can ba put into a particularly convenient form to study the effect of inhomeneous density distributions, such as might arise, for example, in tapering the plasma in wake field accelerator schemes. Here, we shall restrict our attention to the case of uniform unperturbed clensity, so that the wake can be put in the form of a sum of harmonic waves, namely

$$
\frac{n}{n_{0}}=\sum_{m} \sum_{n} i^{n} J_{m-n}(m V) J_{n}[m \omega(H, V) H] e^{i m \omega(H, V)(t-z)}
$$

The use of the weakly relativistic approximation for the electron oscillation, Eq. (10), means that Eq.(31) is valid where the sum over $m$ is dominated by the small $m$.

Consider now the case of highly relativistic electrons descibed by Eq. (S). Here, it appears that the wake structure can be expressed as an implicit function of $(t-z)$. Rather than using the harmonic representation of the Dirac distribution in Eq. (29), we first make use of the representation

$$
n(z, t)=\int d z_{0} n\left(z_{0}\right) \delta\left[z-z_{0}-h\left(t, z_{0}\right)\right]=n_{0}\left[1+\frac{\partial h}{\partial z_{0}}\right]^{-1} .
$$

In order to simplify we retain only zero order plasma effects inside the pulse, namely, we use $H=h_{0}$ and $V=0$. The Lagrangian compression can then be calculated with the help of the chain rule for differentiation applied to Eq. (8). After some algebra, one obtain

$$
\frac{n}{n_{0}}=\left[1-\frac{2 \omega H \operatorname{cn}(\omega \tau, k) \operatorname{dn}(\omega \tau, k)}{2+\omega_{p}^{2} H^{2} \mathrm{cn}^{2}(\omega \tau, k)}\right]^{-1}
$$


where $\tau$ is an implicit function of $t-z$ given by

$$
t-z=\tau-\frac{K(k)}{\omega}-H \operatorname{sn}(\omega \tau, k)+\frac{\omega_{p}^{2} H^{2}}{2} \int_{\frac{k(k)}{\omega}}^{\tau} \operatorname{cn}^{2}(\omega u, k) d u .
$$

Overtaking between neigboring electrons occurs when the velocity of an oscillating electron reaches the phase velocity of the wave [9]. The overtaking in the Lagrangian picture leads to wavebreaking in the Eulerian picture, which results in the production of fast ele trons. Because we have assumed that the group velocity of the pulse is equal to the velocity of light, overtaking clearly is impossible. Thus, the phenomenon of wavebreaking can not be addressed within the framework of the present model. In fact, in the strongly nonlinear regime, $a \gg 1$, a precise and meaningful definition of the group velocity is still lacking.

We have shown in Sec. 3 that $\gamma+\omega_{p}^{2} h^{2} / 2$ is an invariant, so that, behind the pulse, there is a the maximum electric field $E_{M}$, and a maximum relativistic energy $\gamma_{M}$ of an electron during its nonlinear oscillation in the plasma wave. These quantities are given by

$$
E_{M}^{2}=2 \omega_{p}^{2}\left(\gamma_{M}-1\right)=\omega_{p}^{4} H^{2}+\omega_{p}^{2} \dot{V}^{2}
$$

Note that, here, $E_{M}$ is the peak electric field behind the pulse and should not be confused, in the literature, with the maximum electric field of an infinite nonlinear plasma wave [9], arising from the wavebreaking limit. Although, as mentioned above, the wavebreaking limit is not considered in the present model, it so happens that even if the group velocity of the pulse were smaller than c, Eq. (35) remains valid. This happens because the maximum values of these quantities are reached here just behind the pulse, where we solved only an initial value problem, prior to the completion of a plasma oscillation. On the other hand, the difficulties that arise in overtaking occur only upon the completion of one plasma oscillation, and there arise particular difficulties in studying the steady state . of non-linear plasma waves.

\section{$6 \quad$ Nonlinear collective energy losses}

If one views the pulse as a ballistic perturbation sweeping a one-dimensional array of non-linear oscillators, one can imagine a continuous transfer of energy from this ballistic perturbation to the array of oscillators. The energy balance of such a system can be performed straightforwardly. Compare the plasma energy before and after the pulse passing; the difference is the work of the pulse on the plasma,

$$
\frac{d U}{d t}=-\frac{\omega_{p}^{4}}{2} H^{2}-\frac{\omega_{p}^{2}}{2} V^{2}
$$

The nonlinear character of this dissipation is obvious from the fact that it is proporticnal to the square of the electron density. A linear, resonant dissipative process would incur this loss of energy through a decrease in the amplitude of the wave at constant frequency. 
However, since the processes involved here in this energy exchange are nonresonant, the wave action is conserved, and that the interaction result in a slowing down of the pulse, i.e., a decrease in the mean frequency $\bar{\omega}$.

\section{Nonlinear Compton scattering}

Competing with the losses calculate in Sec. 6, due to a coherent transfer of energy to longitudinal waves, is an incoherent transfer of energy to transverse waves. The latter transfer can dominate at low density. When an electron enters the pulse, it is accelerated in the transverse direction, in the process radiating part of the energy in the pulse. This spontaneous process is, in fact, nonlinear Compton scattering. The single particle dissipation is given by [10]

$$
\frac{d \gamma}{d \tau}=-\frac{2 r_{e}}{3} \gamma\left[\frac{d \mathrm{p}}{d \tau} \cdot \frac{d \mathrm{p}}{d \tau}-\frac{d \gamma}{d \tau} \cdot \frac{d \gamma}{d \tau}\right] .
$$

Neglecting both the plasma response, and the radiation reaction force, inside the pulse we can exploit the fact that $t-z$ is the proper time $\tau$. The total energy loss $S$, resulting from one electron transit through the pulse, can then be put in the form

$$
S=\frac{2 r_{e}}{3} \int_{0}^{T} \dot{a}^{2}(u)\left[1+a^{2}(u) / 2\right] d u .
$$

The bracketed term in the integral above accounts for the nonlinear, relativistic modifications of the usual Thomson cross section. To obtain the pulse energy loss due to Compton scattering, we sum the contributions from all electrons entering the pulse. The additional loss term, which would complete the coherent losses in Eq. (33), is then given by

$$
\frac{d U}{d t}=-\omega_{p}^{2} S .
$$

As might be imagined, these incoherent losse: iend, at very low density, to dominate dissipation due to collective effects.

\section{Application to relativistic harmonic generation}

A short intense pulse produces transverse harmonic fields because of the nonlinear response of the plasma [5]. This occurs in addition to the effects considered above: nonlinear Compton scattering and the generation of a longitudinal wake. In harmonic generation, the polarization of the wave plays an important role. In a linearly polarized pulse, the quantity $a^{2}$ is characterized by two time scales, $2 \omega$, and $\delta \omega ;$ on the other hand, a circularly polarized pulse, in effect, characterizes $a^{2}$ with only one time scale, namely, the 
pulse width, $\delta \omega$. This difference between the circular and linear polarization is depicted schematically in Figs. 1 and 2. Linear polarization, becavise $a^{2}$ contains the time scale 2u, gives rise to harmonic generation at $3 \bar{\omega}$.

The transverse current, due to the electrons response inside the pulse, is given by

$$
\mathrm{j}=-\epsilon_{0} \omega_{p}^{2} \int d z_{0} \delta\left(z-z_{0}-h\right) \frac{\mathrm{a}}{\gamma}=-\epsilon_{0} \omega_{p}^{2} \frac{\mathrm{a}}{\gamma}\left[1+\frac{\partial h}{\partial z_{0}}\right]^{-1} .
$$

The ponderomotive perturbation of the density inside the pulse, $\left(1+\partial h / \partial z_{0}\right)$, can be calculated using the results of Sec. 4. Expanding in the density, the leading terms for the proper time of an electron initially at $z_{0}$ are given by

$$
\tau=t-z_{0}-h-\frac{\omega_{p}^{2}}{2} \int_{0}^{t-z_{0}-h} d u \int_{0}^{u} d v \int_{0}^{v} d s a^{2}(s) .
$$

Now use the identity $\partial h / \partial z_{0}=(d h / d \tau)\left(\partial \tau / \partial z_{0}\right)=p \partial \tau / \partial z_{0}$, to find, after some alge'sra, the density perturbation

$$
\left[1+\frac{\partial h}{\partial z_{0}}\right]^{-1}=\gamma\left[1-\frac{\omega_{p}^{2}}{2} \int_{0}^{\tau} d u \int_{0}^{u} d v a^{2}(v)\right] .
$$

Note that using Eq. (42), the current defined in Eq. (40) appears to be a sum of the usual linear reactive contribution and an active nonlinear contribution. In the case of linear polarization, the nonlinear contribution contains the third harmonic of the original pulse. Let $\mathbf{a}_{1}$ be the harmonic vector potential radiated by this nonlinear part of the current, where $a_{1}$ obeys the inhomogeneous wave equation,

$$
\left[\frac{\partial^{2}}{\partial z^{2}}-\frac{\partial^{2}}{\partial t^{2}}\right] \mathbf{a}_{1}=-\mathbf{a} \frac{\omega_{p}^{4}}{2} \int_{0}^{t-z} d u \int_{0}^{u} d v a^{2}(v)
$$

which can be solved using the Green's function for the one-dimensional wave operator. The Green's function is 0 everywhere except in the backward light cone denoted, in Fig. 3, by $C_{+}$and $C_{-}$. In this causal light cone, the Green's function takes the value $1 / 2$. Thus, to solve Eq. (43), the integral over the Green's function is resticted to the intersection of this light cone with the support of $j_{1}$, shown as the shaded area in Fig. 3. Assume now that the interface between the vacuum and the plasma is located at $z=0$, make a change of variables from $(z, t)$ to $(z-t, z+t)$, whose Jacobian is 2 , and find for the first order plasma induced harmonic pulse

$$
\mathbf{a}_{1}(t, z)=-\frac{\omega_{p}^{4}}{2}(z+t) \int_{0}^{t-z} \mathbf{a}(w) d w \int_{0}^{w} d u \int_{0}^{u} d v a^{2}(v)
$$

This result can be simplified if we write $z+t=2 t-(t-z)$, and then note that the first order harmonic response appears as the sum of a growing propagating term and a 
constant propagating one, i.e., furiction of $(t-z)$ only. After few plasma periods, the constant response is dominated by the growing term. The associated unstable electic field $-\partial\left(\mathbf{a}_{1} / \partial t\right)$ can then bw written as

$$
\frac{\partial \mathbf{a}_{1}}{\partial t}=\omega_{p}^{4} t \mathbf{a}(t-z) \int_{0}^{t-z} d u \int_{0}^{v /} d v a^{2}(v) .
$$

To describe the power conversion due to this unstable term, we introduce the integrated quantities $R$, where

$$
R=\int_{0}^{T} d l a^{2}(l)\left[\int_{0}^{l} d w \int_{0}^{w} d v a^{2}(v)\right]^{2} .
$$

The energy, in the harmonic pulse grows as $\omega_{p}^{8} t^{2} R$, representing a power loss, competing with the terms in Eq. (36), which may be written as

$$
\frac{d U}{d t}=-2 \omega_{p}^{8} t R
$$

The integral 2 captures the information relevant to the harmonic generation effect. Evidentally, the harmonic power conversion scales as $\omega_{p}^{8}$, or with the fourth power of the plasma density.

Note that, by varying the degree of polarization of the original pulse, the mean frequency of the harmonic unstable pulse can be tuned between $\bar{\omega}$ and $3 \bar{\omega}$.

\section{Application to relativistic photon acceleration}

In an optimally efficient scheme for relativistic photon acceleration, a leading pulse, depicted as "P1" in Fig. 4, delivers energy to the plasma wake, and this energy is entirely reabsorbed by a lagging or accelerated pulse, depicted as "P2." Complete pulse reabsorption, leaving no energy in the plasma, implies

$$
H_{1}=H_{2}, \quad V_{1}=V_{2},
$$

where $H_{i}$ and $V_{i}$ are the position and velocity shifts following pulse $i$. Since the energy exchange process does not involve resonant processes, it follows that the pulse action is an adiabatic invariant. Hence, in absorbing the wake energy, the lagging pulse experiences a mean frequency upshift [11], rather than a growth in amplitude.

The salient parameters of such a photon accelerator are the mean frequency upshift of the accelerated pulse and the delay between the two pulses. A precise calculation of the mean frequency upshift requires a study of the adiabatic transfer of energy from an ocillating electron to a pulse, which is beyond the scope of the present study. We can, however, calculate directly the optimally efficient time delay in an underdense plasma.

In the nonrelativistic regime this delay, is simply $n+1 / 2$ times the plasma period, where $n$ is an integer. On the other hand, in the relativistic regime, which is particularly 
important because of the possibility of large density gradients, proper time corrections are needed to calculate this delay.

Consider an underdense plasma, so that the exit velocities $V_{i}$ can be neglected $\left(V_{i}=\right.$ $\left.0, H_{i}=H\right)$. The condition for an efficient transfer of energy then becomes $h(D)=-H$, where $D$ is the electron proper time delay between the two pulses. Using now Eq. ( $($ ), the condition can be put in the form

$$
\omega D=2 n K(k), \quad \omega^{2}=\omega_{p}^{2}\left(1+\omega_{p}^{2} H^{2} / 4\right), \quad k^{2}=\omega_{p}^{4} H^{2} / 4 \omega^{2} .
$$

This condition on the proper time of the electron becomes a condition on the time delay between the two pulses $t(H, n)$, such that

$$
\omega t(H, n)=2 n K(k)+\frac{\omega_{p}^{2} H^{2}}{2} \int_{0}^{2 n K(k)} \operatorname{cn}^{2}(u, k) d u,
$$

which gives the condition for the time delay that allows a photon accelerator, operating in an underdense plasma but in the relativistic wake regime, to be maximally efficient.

\section{Density regimes for dissipation}

The previous Sections shows that, to lowest order in $\omega_{p}$, there are three main channels for non linear energy dissipation, and, for very short pulses $(V=0)$, the total dissipation is

$$
\frac{d U}{d t}=-\frac{\omega_{p}^{4}}{2} H^{2}-\omega_{p}^{2} S-2 \omega_{p}^{8} t R
$$

From Eq. (51), we discern several different density regimes of dissipation. To investigate the boundaries between these regimes, consider, for $a>1$, the scaling of the parameters, $H, S$, and $R$ :

$$
H \sim a^{2} \delta \omega^{-1}, \quad S \sim a^{4} \delta \omega^{-1} \bar{\omega}^{2} r_{e}, \quad R \sim a^{6} \delta \omega^{-5} .
$$

We expect, then, that incoherent spontaneous losses will dominate coherent longitudinal dissipation for densities is low enough that

$$
r_{e}>\left(\frac{\omega_{p}}{\bar{\omega}}\right)^{2}\left(\frac{c}{\delta \omega}\right)
$$

where $r_{e}$ is the classical electron radius. Harmonic generation may dominate wake generation at higher density and higher laser intensity, but here there is an important dependence upon the duration of the process or the length of the plasma. After a time, $t$, the losses due to harmonic generation dominate if

$$
\omega_{p} t>\left(\frac{\delta \omega}{\omega_{p}}\right)^{3}\left(\frac{m c}{e a}\right)^{2} .
$$

At the present state of the art of short, intense laser pulse technology, losses incurred through wake generation always dominate, and losses due to harmonic generation are negligible. 


\section{Summary and discussion}

We have shown in the previous sections that many of the effects of an intense, short laser pulse on a cold, underdense plasma can be calculated by solving the equation of motion of each plasma electron, and then summing the effects of all these motions. The result, valid to all order in the intensity, $a$, gives the collective transverse and longitudinal induced fields. The nonlinear energy losses are mainly due to the longitudinal induced wake, which scales as $\omega_{p}^{4}$. The losses due to the transverse induced response, which happens at high frequency only in the case of non circular polarization, scales as $\omega_{p}^{8}$. In the weakly relativistic regime, these losses are negligible compared to the longitudinal losses. Incoherent nonlinear Compton scattering becomes important only at very low density.

We have been mainly concerned by the effect $c i$ a given pulse on the plasma; what we have not considered in detail is the self-consistent problein that considers also the effect of the plasma on the pulse. We show, however, that these effects occur on a longer time scale than do the effects considered above, and so may be neglected.

Two time scales are associated with the modification of the pulse due to the plasma: one, a nonlinear time scale associated with the energy losses; and, two, a linear time scale associated with the dispersive dynarrics of the pulse envelope. The time scale $t_{L}$ associated with the linear dispersive evolution of the pulse shape arises because of the depence of the group velocity on the frequency, $\delta \omega t_{L} \partial v_{g} / \partial \omega \sim c / \delta \omega$. Using the usual dispersion relation for a cold plasma, we find, $\omega_{p} t_{L} \sim(\bar{\omega} / \delta \omega)^{2}\left(\bar{\omega} / \omega_{p}\right)$. This time scale is larger than the time scales associated with the processes studied above. To evaluaie the nonlinear time scale, $t_{N}$, associated with the longitudinal energy losses, we use the global energy balance, and we find $\omega_{p} t_{N} \sim\left(\bar{\omega} / \omega_{p}\right)^{2}\left(\delta \omega / \omega_{p}\right)$. This time scale is also longer than those associated with the processes studied in this paper.

In summary, on the basis of a fully relativistic Lagrangian density expansion, we have demonstrated that the effects of an ultrashort intense laser pulse on a cold plasma can be captured, in fact, by a small set of integral parameters, H, V, R, S, and U. The general formula for the nonlinear wake, nonlinear Compton losses, and harmonic generation have been obtained in term of these global quantities. In addition, what emerges from these new results, and from the Lagrangian method for obtaining them, is a more clear physical picture of the nonlinear processes involved in the ultrashort, pulse-plasma interaction.

\section{Acknorvledgements}

The authors would like to thank P. Sprangle and E. Valeo for a useful discussion of harmonic generation. This work was supported by the United States Department of Energy, under contract number DE-AC02-76-CHO3073. 


\section{References}

[1] T. Tajima and J.M. Dawson, Phys. Rev. Lett. 43, 267 (1979). S.C. Wilks, J.M. Dawson, W.B. Mori, T. Katsouleas, and M.E. Jones, Phys. Rev. Lett. 62, 2602 (1989). T. Katsouleas, W. B. Mori, J.M. Dawson, and S. Wilks Femtosecond to Nanosecond High Intensity Lasers and Applications S.P.I.E. 1229, 98 (1990).

[2] M. Pessot, J. Squier, G. Mourou and D.J. Harter, Opt. Lett. 14, 797 (1989). M. Pessot, J. Squier, P. Bado, G. Mourou and D.J. Harter, IEEE J. Quantum Electron. QE-25, 61 (1989).

[3] J.H. Eberly, Progress in Optics, North Holland, Amsterdam, 7, 359 (1969). Y.B. Zeldovich Sov. Phys. Usp. 18, 79 (1974).

[4] M.N. Rosenbluth, and C.S. Liu, Phys. Rev. Lett. 29, 701 (1972). C.M. Tang, P. Sprangle, and R.N. Sudan, Phys. Fluids 28, 1974 (1985). C.J. Mckinstrie, and D.W. Forslund, Phys. Fluids 30, 904 (1987). M. Deutch, B. Meerson, and J.E. Golub, Phys. Fluids B7, 1773 (1991).

[5] P. Sprangle, E. Esarey, and A. Ting, Phys. Rev. A 41, 4463 (1990). P. Sprangle, E. Esarey, and A. Ting, Phys. Rev. Lett. 64, 2011 (1990). A. Ting, E. Esarey, and P. Sprangle, Phys. Fluids B 2, 1390 (1990).

[6] P. Kaw and J. Dawson, Phys. Fluids 13, 472 (1969).

[7] J.H. Eberly and A. Sleeper, Phys. Rev. 176, 1570 (1968).

[8] J.M. Dawson, Phys. Rev. 113, 383 (1958). O. Buneman, Phys. Rev. 115, 503 (1959). R.C. Davidson and P.P. Schram, Nuclear Fusion, 8, 183 .(1968).

[9] A.I. Akhiezer, and R.V. Polovin Sov. Phys. JETP 3, 696 (1956). J.M. Dawson, Phys. Rev. 113, 383 (1958). T. Speziale and P.J. Catto, Phys. Fluids 22, 681 (1979). W.B. Mori and T. Katsouleas, Physica Scripta, 30, 127, (1990). S.V. Bulanov, V.I. Kirsanov, and A.S. Sakharov, JETP lett. 53, 565 (1991).

[10] E.S. Sarachik and G.T. Scappert, Phys. Rev. D1 2738 (1970). J.E. Gunn and J.P. Ostriker, Astr. J. 165, 523 (1971). R.E. Waltz and O.P. Manley, Phys. Fluids 21, 808 (1978).

[11] E. Esarey, A. Ting, and P. Sprangle, Phys. Rev. A 42, 3526 (1990). 


\section{Figures}

Fig.1 Space-time diagram of the interaction of an electron initially at $z_{0}$ with a circularly polarized laser pulse. Within the pulse, the electron is deflected by the laser. Upon leaving the pulse, the electron oscillates in the self-consistent, nonlinear plasma wake.

Fig.2 Space-time diagram of the interaction of an electron initially at $z_{0}$ with a linearly polarized laser pulse. Within the pulse, the electron is deflected by the laser, where it oscillates at twice the mean pulse frequency. Upon leaving the pulse, the electron oscillates in the self-consistent, nonlinear plasma wake.

Fig.3 Only events inside the causal cone $\left(C_{+}, C_{-}\right)$can influence the point $(t, z)$. Inside the causal cone, only the shaded area, where the interaction between the incident pulse and the plasma takes place, can support the nonlinear current source, $j_{1}$.

Fig.4 Two pulses, $P_{1}$ and $P_{2}$, such that all the energy transferred from pulse $P_{1}$ to the plasma longitudinal relativistic wake is reabsorbed by pulse $P_{2}$ after $1 / 2$ a relativistic plasma period. 


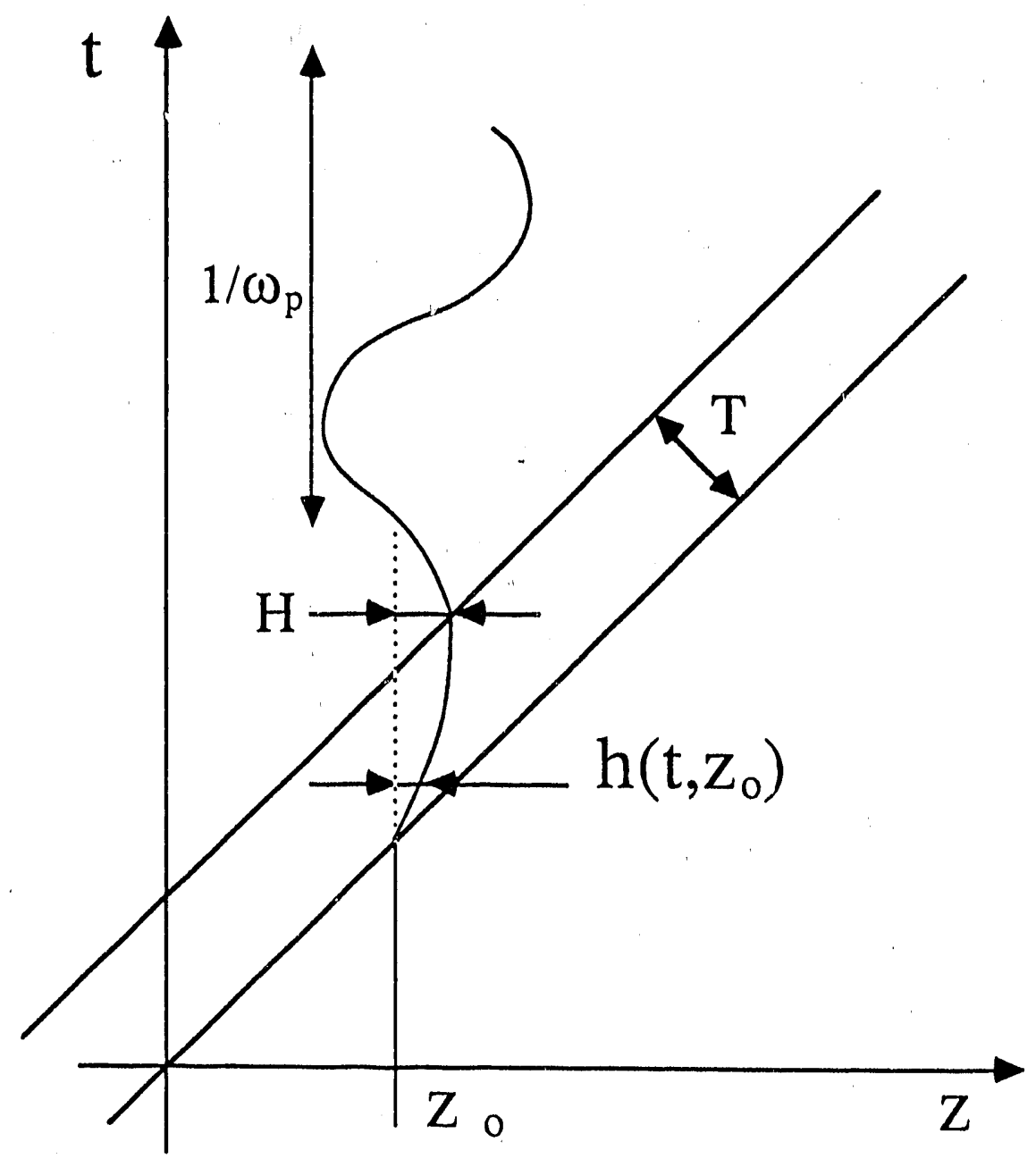

Fig. 1 


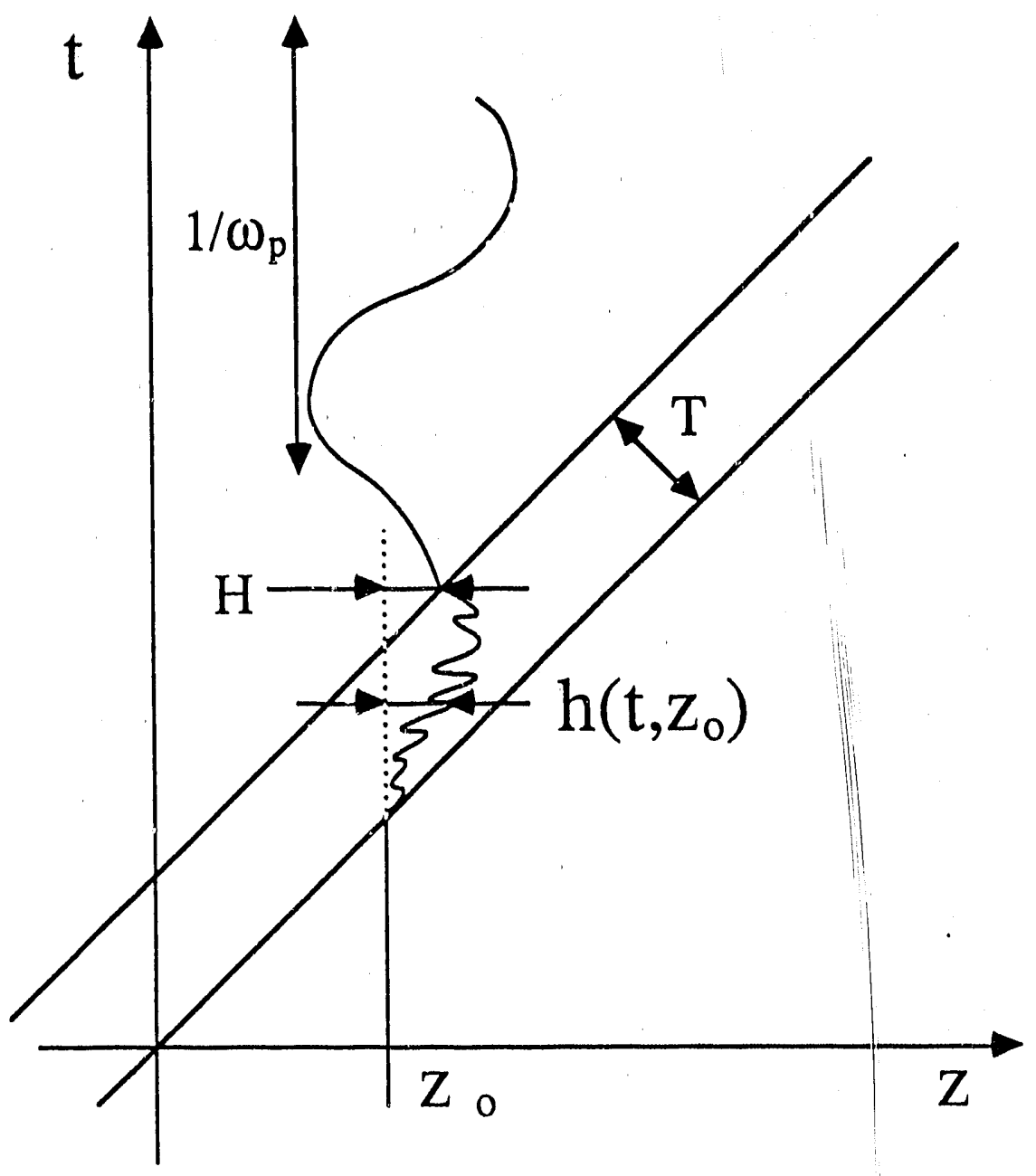

Fig. 2 


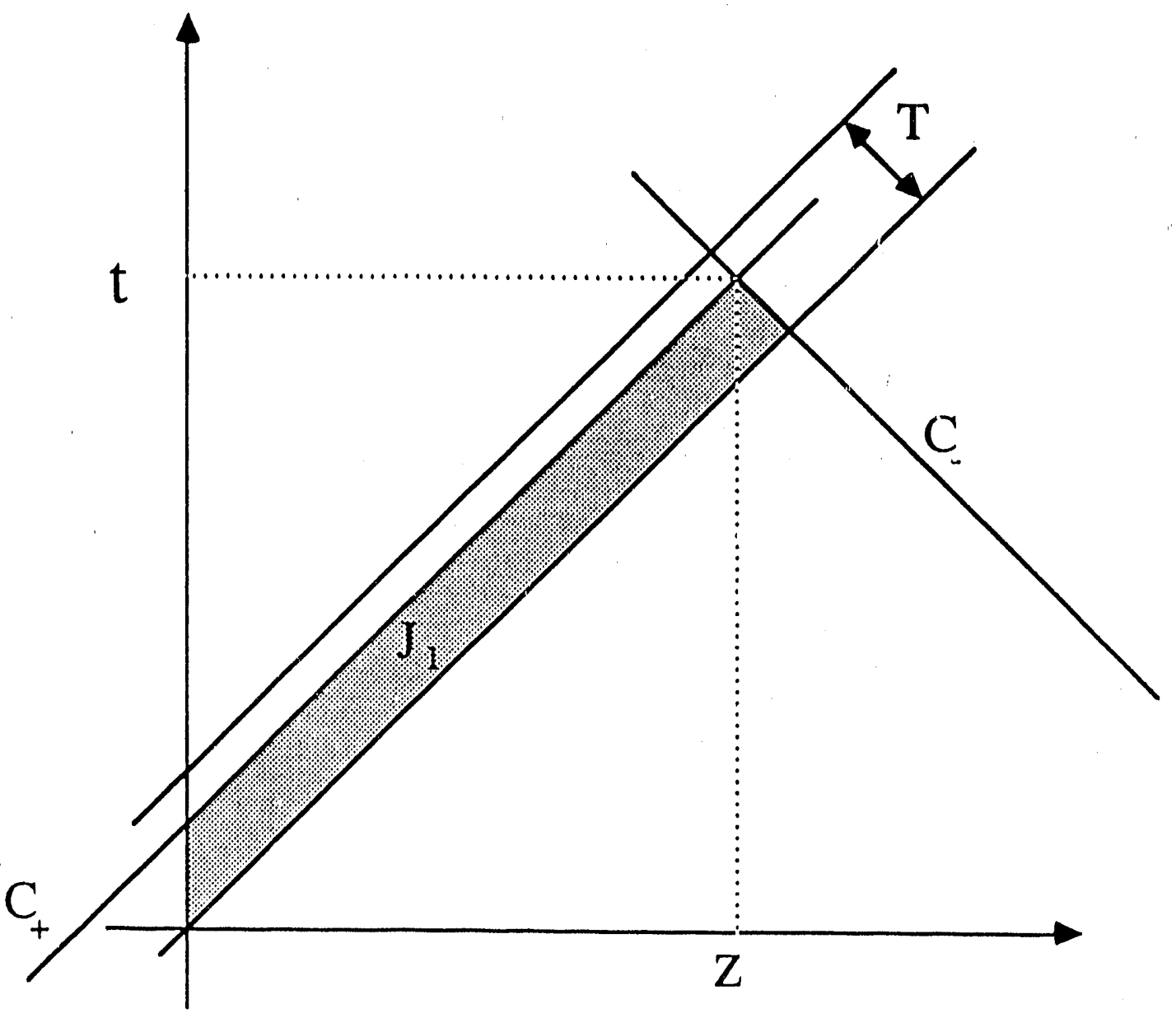

Fig. 3 


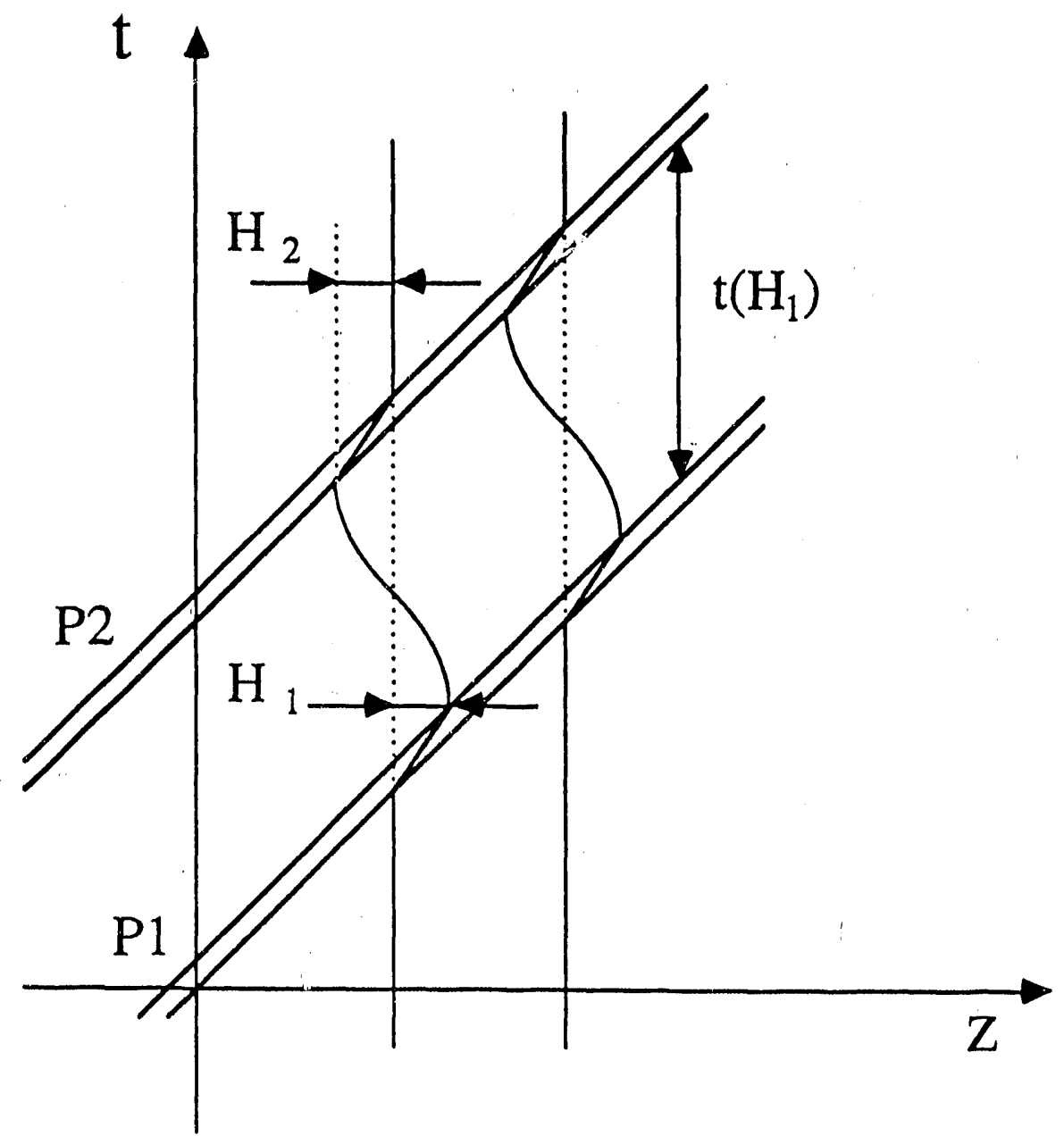

Fig. 4 


\section{EXTTERNAL DISTRIBUTION IN ADDITION TO UC-420}

Dr. F. Paoloni, Univ. of Wollongong, AUSTRALIA

Prof. M.H. Erennan, Univ. of Sydney, AUSTRALIA

Plasma Research Lab., Australian Nat. Univ., AUSTRALIA

Prof. I.R. Jones, Filinders Univ, AUSTRALIA.

Prof. F. Cap, Inst. for Theoretical Physics, Al/STRIA

Prof. M. Heindler, Institut for Theoretische Physik, AUSTRIA

Prof. M. Goossens, Astronomisch Instituut, BELGIUM

Ecole Royale Militaire, Lab. de Phy. Plasmes, BELGIUM

Commission-European, DG. XII-Fusion Prog., BELGIUM

Prof. A. Boudquie, Rijksuniversiteli sent, BELGIUM

Dr. F.H. Sakanaka, Instituto Fisica, BRAZIL

Instiusto De Pesquisas Espaciais-INPE, BRAZIL

Documents Office, Atomic Energy of Canada Lid., CANADA

Dr. M.P. Bechynskj, MPB Technologies, Inc., CANADA

Dr. H.M. Skarsgard, Univ. of Saskatchewan, CANADA

Prof. J. Toichmann, Univ. of Montroal, CANADA

Prof. S.R. Sreonivasan, Univ. of Calgary, CANADA

Prof. T.W. Johnstion, INRS-Energie, CANADA

Dr. R. Bolton, Centre canadien de tusion magnétique, CANADA

Dr. C.R. James, Univ. of Alberta, CANADA

Dr. P. Lukac, Komenskeho Universzita, CZECHOSLOVAKIA

The Librarian, Cutham Laborationy, ENGLAND

Library, R61, Rutherford Appleton Laboratory, ENGLAND

Mrs. S.A. Hutchinson, JET Library, ENGLAND

P. Măhönen, Univ. of Helsinki, FINLAND

C. Moutret, Lab. de Physique des Milieux lonisés, FRANCE

J. Radot, CEN/CADARACHE - Bat 506, FRANCE

Ms. C. Rinni, Univ. of loannina, GREECE

Dr. T. Mual, Academy Bibliographic Ser., HONG KONG

Preprint Library, Hungarian Academy of Sci., HUNGARY

Dr. B. Das Gupta, Saha Inst. of Nuclear Physics, INDIA

Dr. P. Kaw, Inst. for Plasma Research, INDIA

Dr. P. Rosenau, Israel inst. of Technology, ISRAEL

Librarian, Intemational Center for Theo Physics, ITALY

Miss C. De Palo, Associazione EURATOM-ENEA, ITALY

Dr. G. Grosso, Istituto di Fisica del Plasma, ITALY

Dr. H. Yamato, Toshiba Res \& Devel Center, JAPAN

Prof. I. Kawakami, Atomic Energy Res. Inst., JAPAN

Prof. K. Nishikawa, Hiroshima Univ., JAPAN
Director, Japan Atomic Energy Research Inst., JAPAN

Prof. S. Itoh, Kyushu Univ., JAPAN

Data and Planning Center, Nagoya Univ., JAPAN

Prof. o. Tanaka, Kyoto Univ., JAPAN

Library, Kyoto Univ., JAPAN

Prof. N. Inoue, Univ. of Tokyo, JAPAN

S. Mori, Technical Advisor, JAERI, JAPAN

O. Mitara, Kumamoto Inst. of Tochnology, JAPAN

H. Jeong, Korea Advanced Energy Research Inst., KOREA

Prof. D.I. Choi, The Korea Adv, Inst. of Sa. \& Tech., KOREA

Prof. B.S. Liley, Univ. of Waikato, NEW ZEALAND

Inst. of Plnsma Physics, PEOPLE'S REPUBLIC OF CHINA

Librarian, Inst. of Physics, PEOPLE'S REPUBLC OF CHINA

Library, Tsinghua Univ., PEOPLE'S REPUBLIC OF CHINA

Z. LI, S.W. Inst Physics, PEOPLE'S REPUBLIC OF CHINA

Prof. J.A.C. Cabral, Instituto Superior Tecrico, PORTUGAL

Dr. O. Petrus, ALI CUzA Univ., nOMANIA

Dr. J. do Villiers, Fusion Studies, AEC, S. AFRICA

Prof. M.A. Hellberg, Univ. of Natal, S. AFRICA

C.I.E.M.A.T, Fusion Division Library, SPAIN

Dr. L. Stentlo, Univ. of UMEA, SWEDEN

Library, Royal Inst. of Technology, SWEDEN

Prot. H. Wilhelmson, Chalmers Univ. of Tech., SWEDEN

Centre Phys. Des Plasmas, Ecole Polytoch, SWITZERLAND

Bibliotheok, Inst. Voor Plasma-Fysica, THE NETHERLANDS

M. Durgut, Vice Chairman, Middle East Tech. Univ., TURKEY

Dr. D.D. Ryutov, Siberian Branch of Academy of Sci., USSR

Or. G.A. Ellseov, Kurchatov Inst., USSP

Librarian, The Ukr.SSR Academy of Sciences, USSR

Dr. L.M. Kovrizhnykh, Inst. of Generad Physics, USSR

Kerntorschungsanlage GmbH, Zentralbibliothek, W. GERMANY

Bibliothek, Inst. Fur Plasmaforschung, W. GERMANY

Prof. K. Schindler, Ruhr-Universitát Bochum, W. GERMANY

Dr. F. Wagner, (ASDEX), Max-Planck-Institut, W. GEFMANY

Librarian, Max-Planck-institut, W. GERMANY

Prof. A.K. Janev, Inst. of Physics, YUGOSLAVIA 

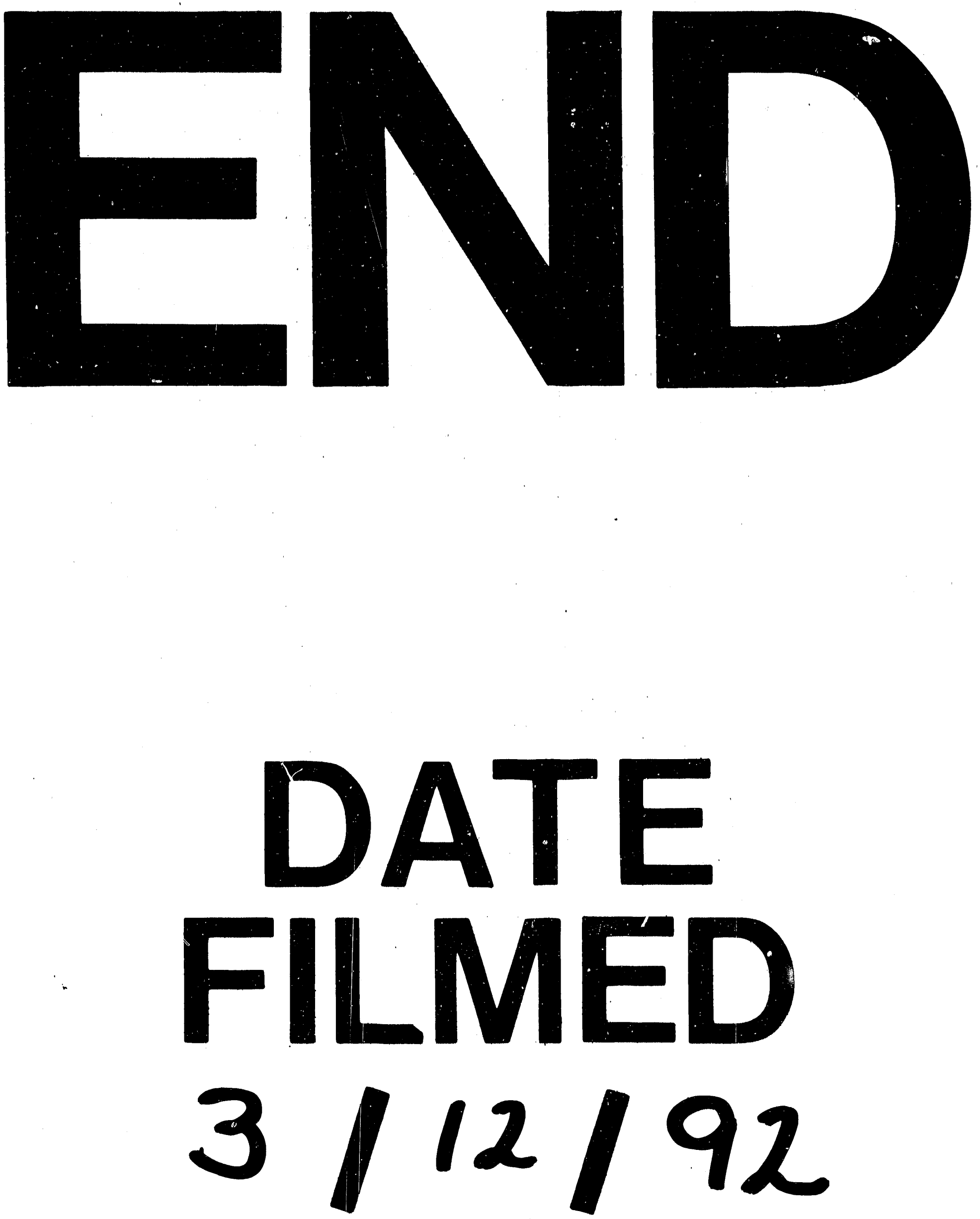

I 
